Stringham, R. Low Mass 1.6 MHz Sonofusion Reactor. in Eleventh International Conference on Condensed Matter Nuclear Science. 2004. Marseille, France.

\title{
Low Mass 1.6 MHz Sonofusion Reactor
}

\author{
Roger Stringham \\ First Gate Energies - PO Box 1230, Kilauea, HI 96754 - firstgate@earthlink.net
}

\begin{abstract}
We are using one of the most remarkable pulsing systems that nature offers for producing transient high energy densities and I have been fortunate enough to be involved with it for over 20 years. Over time we have increased the frequency of our piezo cavitation drivers and are now at $1.6 \mathrm{MHz}$ and find that our results are the same. Even better, the Qx /(reactor gm), the energy density, is drastically increased when compared to our 40 and $20 \mathrm{KHz}$ piezo systems $[1,2,3]$. The cost is decreased by at least an order of magnitude and the durability is greatly increased. All Q values in this paper are $\mathrm{dQ} / \mathrm{dt}$ Joules/sec. or watts. The systems differ in several ways because of the 40 times increase in frequency. These $1.6 \mathrm{MHz}$ systems produce more sonoluminescence, SL, and more but smaller bubbles and an energy density in the collapsing bubble system that is the same magnitude as the $40 \mathrm{KHz}$ systems [4,5]. In one cycle those small bubbles, initially a few hundred $\mathrm{nm}$ in diameter, that are resonance size for the $1.6 \mathrm{MHz}$ input will grow isothermally. After the acoustic wave passes into its positive pressure phase the bubbles collapse violently keeping a portion of their energy. In the final stage of collapse the energy densities are literally astronomical. The collapse process produces from the bubble a jet that implants deuterons into a target foil. The time frame for this $1.6 \mathrm{MHz}$ system is 40 times faster than for the $40 \mathrm{KHz}$ system. The number of deuterons (protons) in the jet drops from $10^{9}$ to $10^{5}$ but the deuteron high density remains the same. The $1.6 \mathrm{MHz}$ low mass, LM, device (weighing $20 \mathrm{gm}$ ) produces the same excess heat, Qx, as the $40 \mathrm{KHz}$ system (weighing $3 \mathrm{Kgm}$ ). The calorimetry is

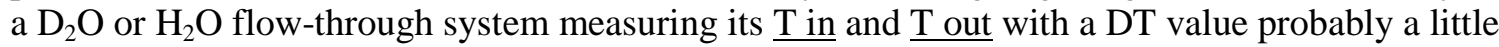
lower than the true value. The flow of $\mathrm{D}_{2} \mathrm{O}$ is measured at $60 \mathrm{ml} / \mathrm{min}$. or $1 \mathrm{ml} / \mathrm{sec}$. The total errors in the Qx measurements are in the order of 2 watts. These values range up to 40 watts depending on acoustic input, temperature, pressure, cavitating liquid and target.
\end{abstract}

\section{INTRODUCTION}

Over 15 years ago in 1989 I heard about the experimental work by Fleischmann and Pons at the University of Utah and within a few days I was using cavitating D2O with a Pd foil target. I saw evidence of foil melting and possible excess heat generation. Three years later after filing patents, the initial three individuals, Dick Raymond, Larry Klein, and myself invited Russ George and Steve Wolff with help from Tom Benson and together started EQuest Sciences. After a year or so we produced helium four, tritium, and Qx. In 1998 EQuest was dissolved and transformed into First Gate Energies with Dick Raymond still president. We were producing excess heat using a $40 \mathrm{KHz}$ system in amounts very similar to what we are producing today with our LM 1.6 MHz device.[1]

We moved our laboratory from Mountain View, CA to Kilauea, Kauai, HI in 2002. Most of our efforts here have been directed to the LM 1.6 MHz sonofusion device. We have limited resources so have lowered our goals to cutting costs and doing calorimetry experiments with the measurement of Qx production. At this time we cannot afford to look for the products of Qx production - the expensive analysis of helium and associated products. These products have been found in the past $[2,3]$ in our lower frequency reactors. The investigation was undertaken to advance sonofusion technology and to find better and more compelling data showing Qx 
production. 1) There are more transient cavitation bubbles, TCBs, formed/sec. 2) Smaller TCB bubbles are produced. 3) The smaller bubbles have less energy but the same energy density at the final stage of the collapse process. 4) There is less target damage due to reduced number of deuterons in the implanting jet. 5) The advantage of incorporating the sonoluminescence, SL, emission data from the sonofusion reactor is its application as a tool. The SL emission is coincidental with the jet plasma formation and deuteron concentration that is implanted into the target.

So we have accomplished the above and found several more advantages using the higher 1.6 $\mathrm{MHz}$ frequency. 1) The data is much more reproducible. 2) The Qx generation is now commercially competitive. 3) These small LM devices can be ganged together resulting in a large and cost effective high-density energy source. 4) Our confidence level in our technology has greatly increased.

The physical phenomena of the TCB and stable cavitation bubble growth and collapse has been well documented [4] but not understood in the physics sense. When compared to the 20 and 40 $\mathrm{KHz}$ systems the short time frame of $0.6 \mu \mathrm{sec}$. for the $1.6 \mathrm{MHz}$ frequency that includes the bubble's complex growth and collapse mechanism is scaled to fit [5]. The path of the birth and death of a TCB in the cavitating liquid during one cycle of $1.6 \mathrm{MHz}$ pressure swing, going from low pressure involving the rapid bubble expansion into the high pressure acoustic compression, follows. The initial infant bubble that is selected by the system's parameters to become a TCB for one acoustic cycle is in a parameter controlled environment. The parameters that control the TCBs in the cavitating water are temperature, pressure and Qa input. See figure 1. Initially among all the bubbles in the low pressure zone is the infant TCB bubble population of resonant size which couples with the $1.6 \mathrm{MHz}$ acoustic input, Qa, of an appropriate voltage. With the temperature and pressure of the water and Qa working together in the LM SF reactor and piezo creates an operating resonance. See figure 1 . The infant bubble grows rapidly isothermally and collapses as a TCB during one acoustic cycle. In a $1.6 \mathrm{MHz}$ time frame the collapse is much more rapid and potentially more energetic but with perhaps one ten thousandth the particles $\left(10^{5}\right.$ particles in the implanting jet). The energy densities are at least the same as the $20 \mathrm{KHz}$ systems and the jet formation is assumed to be similar along with the target implantation $[1,2,3]$. These phenomena are controlled by these three parameters.

There are several scenarios that describe the bubble collapse and the SL emission and several physical dilemmas that do not fit. For example the SL pulse length and the relation between the SL emitting cavity and its wavelength are two of these dilemmas [6]. It is clear that in our experiments we see a correlation between Qx and SL emission. See figure $4 \mathrm{a} \& \mathrm{~b}$. The temperature factor that we have worked with to this point is from 25 to $80{ }^{\circ} \mathrm{C}$. The pressure of $\mathrm{Ar}$ is one atmosphere saturation and there are 4 atmospheres of pressure developed in the reactor via the restricted water flow through the small reactor orifices. There was more than a ten fold increase in SL when the initial Ar was increased from 1 to 2 atmospheres with no corresponding increase in Qx. This is very interesting and is explained by the Ar concentration being responsible for SL not Qx. So to use SL as a tool we must consider the Ar concentration levels which should be the same in Qx comparison studies. We want to engineer the LM SF reactor by doing some material improvements and expect to operate the LM SF reactor at higher temperatures.

\section{CALORIMETRY}

The calorimetry is simple and conservative. We look at the data generated at steadystate temperature conditions with the Qi pulsed one minute on and one minute off to help clarify the magnitude of the radio frequency, RF, interference during the thermocouple measurements. The contribution of RF to the temperature measurements of $\underline{\mathrm{T}}$ in and Tout was at most +0.2 of a 
degree $\mathrm{C}$. The effect is more pronounced in the TC for the oscillator and transformer, O\&T, calorimetry with about $0.5^{\circ} \mathrm{C}$ increase. The DT ( $\mathrm{T}$ out - T in) measurement is of the circulating water through the low mass sonofusion (LM SF) reactor. The other half of the calorimetry measurement is the water flow rate through the $1 \mathrm{ml}$ volume of the LM SF reactor. The flow meter was placed just before the bubbler and regularly monitored (See figure 2). The flow rate values for all runs were between 54 and $60 \mathrm{ml} / \mathrm{min}$. A volume calibration of the flow rate of 54 $\mathrm{ml} / \mathrm{min}$ (measured at the bubbler) show that the volume of water passed $/ \mathrm{min}$ is $60.1 \mathrm{ml} / \mathrm{min}$. This is $12 \%$ higher than the flow-meter measured value at a flow of $54 \mathrm{ml} / \mathrm{min}$. For example the

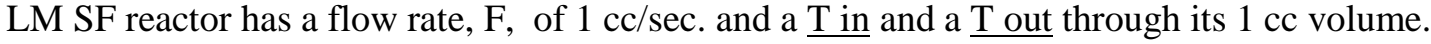
DT $x$ F $x \mathrm{k}=$ Qo and is the basis for our calorimetry.

Prior to each run the circulation system was turned on and $\mathrm{T}$ in and $\mathrm{T}$ out were measured and their values were steady and nearly equal. Then the pulsing acoustic oscillator was turned on (duty cycle) and the data gathering was continued to the end of the run. Data reduction using the collected wattmeter and TC data produces the following calorimetric relations. All Q values in this paper represent $\mathrm{dQ} / \mathrm{dt}$ Joules/sec. or watts.

Qi = watts in from wattmeter

Qo\&t $=$ the total watts lost to the oscillator box calorimetry with Joule heater, JH, for calibration $\mathrm{Qa}=\mathrm{Qi}-\mathrm{Qo} \& \mathrm{t}$ the acoustic watts into reactor

$\mathrm{Qo}=$ the total watts out of reactor

$\mathrm{Qx}=\mathrm{Qo}-\mathrm{Qa}$ the watts of excess heat.

The total watts out, Qo, equals Qa plus Qx where Qx = DTxFx4.184 - Qa, and where F is the flow rate in $\mathrm{ml} / \mathrm{sec}$. The acoustic watts input, $\mathrm{Qa}$, is determined from a Lucite closed box calorimeter inside the air circulating light box which houses the reactor and PMT, photomultiplier. The Lucite calorimetry box contains the oscillator and transformer, O\&T, and is calibrated against the input of a 15 watt Joule heater to find the steadystate heat lost to the O\&T. The heat lost by the O\&T is Qo\&t and is that portion of Qi that is not Qa. A linear plot of JH watts-input vs the calorimeter steadystate DT for O\&T is used to determine Qo\&t values for Qi. The total input, Qi, was divided between Qo\&t and Qa and relates to the efficiency, E, of the acoustic input, the ratio of Qa/Qi. E varied between .33 and .23 for these experiments. The time for the transformer to reach steadystate was 3 hours because of its high mass. This required more experimental time. The time for the LM sonofusion reactor to reach a practical steadystate was five minutes.

For example, a LM SF reactor weighing $20 \mathrm{gm}$ generates data for its calorimetry at steadystate. The reactor has a mass water flow through it of $1 \mathrm{ml} / \mathrm{sec}$ and with a DT of $15^{\circ} \mathrm{C}$. From DT* $\mathrm{F}^{*} \mathrm{k}$ we calculate the Qo $=$ of 55 watts. We know from Qo\&t and $\mathrm{E}$ how to partition a 50 watt Qi that determines the value of Qa. Subtract 35 watts, the calorimetric value of Qo\&t, from 50 watts Qi to give the 15 watts for Qa. Subtracting Qa from Qo leaves a Qx of 40 watts.

The analysis of the data shows the importance of Newton's Cooling Law when applied to the heating and cooling of water flowing through the LM SF reactor. The matching cooling and heating curves $\left(\mathrm{T}=\mathrm{T} \operatorname{sse} \mathrm{e}^{-\alpha \mathrm{A} / \mathrm{CM}}\right.$ and $\mathrm{T}=\mathrm{Tss}\left(1-\mathrm{e}^{-\alpha \mathrm{At} / \mathrm{CM}}\right)$ or $\left.\mathrm{T}=\mathrm{Tss} \mathrm{e}^{-\mathrm{kt}}\right)$ seen in the data are the result of the pulsed acoustic input into circulating water. (Being pulsed, the reactor system does not reach a final steadystate but cycles between a high and a low value approaching its steadystate temperature value.) The constant $k$ that is the exponent $(\alpha \mathrm{A} / \mathrm{CM})$ can be determined from the data where $\alpha$ is the heat transfer coefficient, $\mathrm{A}$ is the surface area of the reactor, $\mathrm{C}$ is the reactor's heat capacity, and $\mathrm{M}$ is the reactor's mass. The Tss is the steadystate temperature and $\mathrm{T}$ is any temperature on that particular heating curve. The heating and cooling curves, $\mathrm{H} \& \mathrm{C}$, can be 
constructed mathematically that closely match the curves produced by the data. One could justify the use of that constructed Tss in the DT calculation. This would add around $15 \%$ to the DT values determined in the pulsing mode. (In the pulsing mode the heating curve reaches a point well up on the heating curve but is cut off by the duty cycle before reaching its true steadystate, Tss. The pulsed low mass, LM, system comes close to a steady state temperature and we use that conservative value for the Qo calculation. In these experiments most of the heated mass is not the LM SF reactor. Most of the heat content is from the mass of water flowing at $1 \mathrm{ml} / \mathrm{sec}$ at its DT. The remaining mass of $20 \mathrm{gm}$. is that of the LM reactor (the momentary container of circulating water) has a 3 times longer time constant for its $\mathrm{H} \& \mathrm{C}$ curves than the circulating water. The $\mathrm{H} \& \mathrm{C}$ curves due to the $\mathrm{LM}$ reactor can be seen in the data with the circulation off. To maintain a DT of $10^{\circ} \mathrm{C}$ for the $20 \mathrm{gm}$ reactor one looks at the initial slope (DT/time) from the cooling curve data of the $20 \mathrm{gm} \mathrm{LM} \mathrm{SF}$ reactor. And from the $\mathrm{H} \& \mathrm{C}$ curves it requires $1 \mathrm{watt}$ input to maintain a DT of $10^{\circ} \mathrm{C}$ in the static reactor. When one looks at the pulsed data of a $10^{\circ} \mathrm{C}$ DT of circulating water that produces 42 watts of Qo (DTxFxk) that number overwhelms the reactor's one watt maintenance value. (The LM SF reactor with several smaller H\&C curves with their associated mass, heat capacity, and longer time constant are used in the above calculation).

Q is the heat in joules/sec or watts for the values below. The error for a typical Qo measurement of 50 watts where watts $=\mathrm{DT} x \mathrm{Fxk}$ is as follows. To produce DT from the input Qi from the wattmeter and the measurement of Qa by the subtraction of Qo\&t from Qi is the path to Qa and the DT determination. The errors in the wattmeter measurement were in the order of \pm 1 watt in 100 watts and in the Qo\&t calorimetry measurement \pm 2 watts and in the TCs $\pm 0.2^{\circ} \mathrm{C}$ in $100{ }^{\circ} \mathrm{C}$. The calculated error in Qa input and DT is then $\pm 7 \%$. The error in the calibrated flowmeter measurement is $\pm 2 \mathrm{cc} / \mathrm{sec}$ for $60 \mathrm{cc} / \mathrm{sec}$ is $3.3 \%$. So the error for Qo is $\pm 8 \%$. The calculation of Qx is Qo - Qa and the error for Qx measurement is $\pm 10.4 \%$. The measurements are on the low side for Qx because of unaccounted convection heat losses to the environment during the experiment which increase as DT increases so the measurements of Qo and Qos are conservative. These are main sources of error.

\section{EXPERIMENTAL}

Water was circulated through the sonofusion reactor's $1 \mathrm{ml}$ volume at a rate of $1 \mathrm{ml} / \mathrm{sec}$. using an FMI pump with a $.635 \mathrm{~cm}$ ceramic piston and sleeve. The cavitation liquid was circulated via the pump through to the $.382 \mathrm{~cm}$ inside diam. stainless steel cooling coil $150 \mathrm{~cm}$ long exchanging the circulating water's heat and exchanging it to an ambient 2 liter coolant water bath. The water is then passed through a $10 \mu$ filter then to the $0.05 \mathrm{~cm}$ inside diam. input reactor orifice (input $\underline{\mathrm{T}}$ in measured with a thermocouple). A residence time for the circulating reactor water of $1 \mathrm{sec}$. to produce the DT of 0 to $12^{\circ} \mathrm{C}$ and with a Qa of 0 to 15 watts means a Qx from 0 to 40 plus watts with the $100 \mu \mathrm{Pd}$ target foil and $\mathrm{D}_{2} \mathrm{O}$ (system A). The hot water left the reactor through a 0.05 $\mathrm{cm}$ inside diam. output reactor orifice (output T out measured with a TC) and out of the light box and into the flow meter. See figure 2. The circulation continues to the Ar bubbler then back to the pump. We used two different target foils, Pd $100 \mu$ thick with cavitating D2O with $1 \mathrm{~atm}$. Ar (system $\mathrm{A}$ ) and $\mathrm{CuBe}(98.1 / 1.9) 125 \mu$ thick with cavitating $\mathrm{H} 2 \mathrm{O}$ with $1 \mathrm{~atm}$. Ar (systemB) which produced about 1/8 Qx of the A system. Both systems were pulsed with a duty cycle of 1 minute on and 1 minute off.

The Qi power to the O\&T was regulated by a variac from 30 volts that produced no SL (only background counts of 100/sec.) to 140 volts with $90 \mathrm{Kcounts} / \mathrm{sec}$ of SL emission ((Kcounts are 1000 counts/sec. registered by the Systron - Donner counter/timer from the Hamamatsu 3 HC 125 03 PMT. See figure 2. The $2 \mathrm{~cm}$ diameter sealed Lexan window of the sonofusion reactor was $90 \%$ covered with $1 \times 2 \mathrm{~cm}$ target foil between the PMT and piezo. The target foil was placed 1 $\mathrm{mm}$ in front of the window and $2 \mathrm{~mm}$ in front of the piezo in the $1 \mathrm{ml}$ of reactor liquid. Even 
with the small volume and foil blockage of the window a maximum of $100 \mathrm{Kcounts} / \mathrm{sec}$ was observed in system B. The SL Kcounts increased with the increase of Qi from the variac voltage. The counter registered a count rate for each second of cavitation foil exposure. It was pointed out that the count was only a relative photon emission rate [6] as photons are emitted in 100 pico second pulses that swamp the PMT. The SL was the only connection we had to the high-speed transient phenomena occurring in the TCB collapse process. The relation of SL and Qx gave us an observational tool to the high density low energy plasma and deuteron or proton production in the $1.6 \mathrm{MHz}$ sonofusion reactor. This was a great aid in adjusting the critical parameters of temperature, Ar pressure, and acoustic input for Qx reproducibility.

The input temperatures for these experiments were steadystate temperatures at $\mathrm{T}$ in which was near ambient. There were some complications from a contribution from $\mathrm{H} \& \mathrm{C}$ curves other than water and are discussed in the calorimetry section. T out was controlled by Qa and Qx, which produced Qo, so it varied from near ambient to 15 degrees above depending on Qi. The running steadystate temperature was at $\mathrm{T}$ out of the sonofusion reactor and the heat disbursing system. The temperatures were measured with TT-K- 40 thermocouples and the data logged by an Omega - Measurement Computing - PCI-DAS-TC with 16 TC channels. There were two types of runs, pulsed and continuous. The pulsed data was naturally cooler being in the on-mode for half the time. The continuous wave experiments agree with the pulsed runs but we are not sure about the possible RF interference. See figure 3. The Ar pressure was kept at 1 atm. by the slow bubbling of $\mathrm{Ar}$ into the $\mathrm{D} 2 \mathrm{O}$ or $\mathrm{H} 2 \mathrm{O}$ bubbler, see figure - 2, and this was not changed during the experiments so the external pressure was always $1 \mathrm{~atm}$. Ar for all runs. The parameter that controlled the Qx production was Qa/Qi. The input from the line voltage was controlled by the variac and passed this power through a wattmeter, a 100 watt Ohio Semitronix Wattmeter, and into a transformer with 50 volt RMS output to a 70 DC volt rail feeding a $1.6 \mathrm{MHz}$ oscillator which produces the Qa powering the reactor piezo. The oscillator and transformer, O\&T, were in a Lucite calorimetry box along with the calibration JH where the Qo\&t losses were determined. The Qa and E could then be determined. See calorimetry section. The Qi is varied via a variac from 4 to 50 watts (the SL threshold is 4 watts). In system A, when Qi is adjusted to 50 watts, the SL is 100,000 counts/sec. and in system B 10,000 counts/sec.[6,8]. The character of Qa produced from our oscillator used in these experiments included a varying voltage of the $1.6 \mathrm{MHz}$ signal carried in a $120 \mathrm{~Hz}$ envelope. The maximum voltage amplitudes of a 50 watt Qi (150 volts peak to peak that includes a family of lesser voltages) produces a maximum Qa at 15 watts. This allows, as we have done earlier experiments, a voltage amplitude that varies from 0 to the maximum voltage for a particular Qi setting. The Qa input of a multi voltage system shows the mechanical resonance of the system to choose its coupling voltage even though it may be small. The Qa maximum signal can be varied from 0 to about 150 volts peak to peak. The data shows that at Qi of 4 watts there is no SL and no apparent Qx above the error level. By increasing Qi to the oscillator from this 4 watt point there is an increasing SL and Qx.

The reactor system was dynamic; the water circulated through the reactor at a measured rate, and differed from a static reactor system where the water remains in the reactor without movement. In the static system the heat must be removed by exterior contact with a flowing heat exchange media. We have used both systems in the past and they both produced Qx. The dynamic system was chosen for its quick response to heat removal. We used the SDS 200, a $200 \mathrm{MHz}$ oscilloscope, with a $5 \mathrm{GS} / \mathrm{sec}$ sampling rate to observe the $1.6 \mathrm{MHz}$ input signal Qa and the SL PMT voltages. The PMT output voltage response was split with a SL event counter that measured the rate of photon emission from the LM SF reactor and oscilloscope. 


\section{RESULTS}

There were two systems studied. System A was the $\mathrm{D}_{2} \mathrm{O}, \mathrm{Pd}$, Ar and system B was the $\mathrm{H}_{2} \mathrm{O}$, $\mathrm{CuBe}, \mathrm{Ar}$. The water circulation was basically $1 \mathrm{ml} / \mathrm{sec}$. and the external pressure of Ar was 1 atmosphere. The controlling parameter was the acoustic input Qi that was partitioned between Qa and Qo\&t which determines the E and equals Qa/Qi. The Pd target foil produced the highest Qx/Qa and the $\mathrm{CuBe}(98.1 / 1.9)$ target foil in $\mathrm{H}_{2} \mathrm{O}$ produced 1/8 the Qx of the Pd foil in $\mathrm{D}_{2} \mathrm{O}$. Both foils appeared untouched by exposure to cavitation. This was a good result as both $\mathrm{Cu}$ and Pd suffered damage when exposed to 20 and $40 \mathrm{KHz}$ cavitation processes. The durability of the foils and the low mass of the 1.6 MHz Qx producing systems have the characteristics needed in a commercial system. The low running temperatures between 30 and 45 degrees $\mathrm{C}$ and the low pressures are much less than the 20 and $40 \mathrm{KHz}$ systems with running temperatures well over 100 ${ }^{\circ} \mathrm{C}$.

During the experiments the systems malfunctioned several times primarily when the water circulation system leaked or plugged causing the reactor to overheat and fail. Sometimes we only needed to fix the leak but on several occasions we lost the piezo when its vapor deposited electrode peeled off the surface or the transistor in the oscillator burned out. The reduced data collected from these systems is shown in figures $4 \& 5$.

\section{INTERPRETATION \& CONCLUSIONS}

The advancements of our sonofusion technology over the last year has grown at a rapid rate. It now is plausible to discuss the possibility of sonofusion becoming an economically commercial energy source. Increasing the cavitation frequency has solved several problems that we have encountered in our past work - size, cost, energy density and durability. The size has been reduced from $5 \mathrm{Kgm}$ to $20 \mathrm{gm}$ and the cost from $\$ 15,000$ to $\$ 150$. The excess heat produced per gram of LM SF reactor has been increased about 500 times and the duration life-time of a cavitation exposed target foil has been extended to a year or more but needs more testing to verify this fact.

The relation between Qx and SL appears that they are coupled together for a particular system. The $\mathrm{H}_{2} \mathrm{O}$ systems produce more SL than do the $\mathrm{D}_{2} \mathrm{O}$ systems. So within a particular system SL is a good predictor for Qx. Figures 5a\&b show these relationships and demonstrate the utility of $\mathrm{SL}$ as a tool. A problem with this tool requires the alignment with the PMT and the careful sizing and placement of the target foil. Also the slow rise time, 70 nano seconds, of the PMT response, is not compatible with the pico second pulse time. However, the somewhat obstructed path of the emitted SL photons moderates that problem. An interesting note on one run where the Ar pressure was increased from the usual 1 to 2 atmospheres of Ar produced 10 times more SL but the same Qx $[6,7,8]$. See figures $4 a \& b$. This phenomena was the result of substantial mechanical pressure increases in the LM SF reactor brought about by its small in and out orifices. Here in the reactor the running pressure did not change so the Qx did not change but the amount of $\mathrm{Ar}$ in the $\mathrm{D}_{2} \mathrm{O}$ was doubled and produced a tremendous change in SL emission with no change in the experimental set-up.

Qo is measured by a conservative process of DT* $\mathrm{F}^{*} \mathrm{k}=$ Joules/sec. and when this number is equal to Qa \pm 2 watts then there is no Qx. But if it is larger, which it will be if Qa is more than 4 watts, then Qo $-\mathrm{Qa}=\mathrm{Qx}$ is a significant number between 3 and 40 watts. This number depends on the value of Qa and as it increases so does Qx. When using the oscillator to drive the piezo there was a limit to the Qa produced as the efficiency, E, (Qa/Qi) as Qi pushed the oscillator to failure. To produce more Qx we look to appropriate changes in the controlling parameters that include increase in pressure, 
temperature, and acoustic input. These improvements would involve the different reactor materials and improving the heat removal system. These small LM SF reactors can be ganged together to form a high energy density array producing Qx that can be transformed into more useable forms of energy.

We looked at two different cavitation pulsed systems. The A system, consisted of a Pd target foil, $\mathrm{D}_{2} \mathrm{O}$, and Ar at $1 \mathrm{~atm}$. The $\mathrm{B}$ system, consisted of a CuBe target foil, $\mathrm{H}_{2} \mathrm{O}$, and Ar at $1 \mathrm{~atm}$.. The systems A and B are the same in every aspect except for the water. When the SL emission count from the PMT is recorded the $\mathrm{H}_{2} \mathrm{O}$ is much larger than for $\mathrm{D}_{2} \mathrm{O}$. The A system is deuterated, the $\mathrm{B}$ system is protonated, and the nuclear paths are quite different after the high density low energy plasma target implantation. The implanted deuterons or protons may follow one of these paths $\mathrm{D}+\mathrm{D} \rightarrow \mathrm{He} 4+$ heat or $\mathrm{P}+\mathrm{P} \rightarrow \mathrm{D}+$ heat or $\mathrm{P}+\mathrm{D} \rightarrow \mathrm{T}+$ heat. No long-range radiation was found, only heat indicating some transient coherence in the implanted species $[2,3,7]$. The pulsed data system A shows that as Qa increases so does Qx to a more than a 2/1 ratio (Qx/Qa) at the higher Qi values. System B shows a much lower increase of Qx as Qa is increased although it does appear to be real with a ratio of about $1 / 2$. If one prefers to call system $B$ a zero for a reference value, it gives all the data a foundation as no true zero has been found to date. See figure 5 a.

System A which produces a power multiplication effect, ME, as high as 1.7 becomes a useful tool for showing the economic advantage of the LM SF reactor water heating capabilities. The whole system including the oscillator and reactor are submerged in a tank of moving water. This allows the complete recovery of Qi plus Qx and their conversion into heat. This ME can be expressed as (Qi +Qx)/Qi and is 1.0 with no Qx production with an input Qi of 50 watts, a Qa of 16 watts and a Qx of 38 watts the ME is 1.7. See figure $5 \mathrm{~b}$.

There is a substantial amount of work to be done to make sonofusion a replacement for a hydrocarbon-based economy and we look forward to that day. We have good continuous cavitation data that shows a 4 times better result for A system than for B system. One of the first tasks is finding by-products that are associated with the Qx production to place next to the Qx evidence. We are currently making improvements at a rapid pace in our sonofusion technology. Make the E, oscillator efficiency, a value approaching 1.0 where it is now 0.3 . Increase the Qx/Qa ratio to 10 where it is now 3.0. Find new and better thermoelectric devices, TEDs, for the direct conversion of heat to electricity. Make the reactor more robust through the application of material technology so reactors can endure higher temperatures and pressures. Perform a marriage of steam technology with the sonofusion reactor. Look for target materials that are improvements over what we have today. The above are a few of the areas that we are currently investigating.

We are now developing the idea of ganging the unit LM SF reactors together to form large heating devices. A practical size to build is a 4 unit device that is being tested with higher temperatures and pressures to produce at least 160 watts of Qx with a much more efficient Qa/Qi of $50 \%$ or better. We are developing the calorimetry for this 4 unit system which should have an ME of 2.3. A Qa of 15, a Qi of 30 and a Qo of 50 and for a 4 unit system 120 watts for Qi, 200 watts for Qo, and 60watts for $\mathrm{Qa} \rightarrow \mathrm{ME}$ of 2.2. If we gang 32 of these 4 unit systems together a $10 \mathrm{~K}$ watt device results. See figure 6.

\section{SUMMARY}

We have today an infant technology that will grow at a very fast pace. Sonoluminescence is a fascinating subject and its study forms the basis of the SF technology. A paper [9] that gives many good references on SL of the single bubble can also be applied to the multibubble systems. 
Also see the references of [7]. We can represent the utility of the sonofusion reactor as a device that can act as a power multiplier using the multiplier effect, $\mathrm{ME}$, to measure the advantage over today's power costs. See figure $5 \mathrm{~b}$. There is one piece of important information missing; evidence of the other products associated with Qx production. So we go back to our old data from our $20 \mathrm{KHz}$ system that reported the products of ${ }^{4} \mathrm{He}$ and ${ }^{3} \mathrm{~T}[2]$ and look for some of these products in the new analysis, by Francesco Celani, of our new target foils and we thank him for any results he may find.

The Qx watts/(gm of reactor) is 2 and we expect that improvements will follow. If for example $\mathrm{Qi}=40$ watts and the Qa/Qi is 0.5 and Qx is 40 watts, then the total watts output for one LM sonofusion unit is 80 watts. This $20 \mathrm{gm} \mathrm{LM}$ reactor can be used as a powermultiplier, ME, of value 2.0. If we have four LM reactors working together as a system the total output is 320 watts and 25 of these 4 unit systems working together produces 8,000 watts with a reactor mass of 2 Kgm.. When 1000 units are ganged together we can produce 80,000 watts that would cost $\$ 300 /$ day (\$ 0.17 for a KWH). However, this system has a ME of 2 and the cost for Qi would be $\$ 150 /$ day. With a 1000 unit reactor the Qx that is produced would be worth $\$ 55,000 / \mathrm{yr}$ and the initial cost for a sonofusion reactor might be $\$ 20,000$ making the cost benefit for the first year $\$ 35,000$. If the 40,000 watts for Qi are solar generated, then we are looking at an added initial cost that would be compatible with our sonofusion reactor with long - term dollar savings. The total heat production is Qi + Qx with a cost reduction of 50\% for low-grade heat used in building's utility heating. This is all possible with the sonofusion technology we have today and this technology is continually improving.

I would like to thank the individuals that helped us get this $1.6 \mathrm{MHz}$ system to the point it is in today: Dick Raymond, Richard America, Kip Wallace, Lynn Marsh, Julie Wallace, Ted Mill, Fran Tanzella, and Mike McKubre.

\section{REFERENCES}

[1] R. Stringham, Proceedings of the IEEE Ultras. Intern. Symp., Sendai, Japan, vol. 2, 1107 (Oct. 5-8, 1998).

[2 ] R. S. Stringham, Proceedings of ICCF-8, Lerici (La Spezia), Italy, 299-304 (21-26 May, 2000).

[3 ] R. S. Stringham, Proceedings of ICCF-9, Beijing, China, 323 (19-24 May, 2002).

[4] M. P. Brenner, Sascha Hilgenfeldt, and D. Lohse, Rev. of Mod. Phy.,74,425-484, (2002).

[5] K. R. Weninger, C. G. Camara, and S. J. Putterman, Phys. E. Rev., 63, 016310-1 (2000).

[6] G. Vazquez, C. Camara, S. J. Putterman, and K. Weninger, Phys. Rev. LETT., 88, 19, 197402-1 (2002)

[7 ] R. S. Stringham, Proceedings of ICCF-10, Boston, USA (24-29 Aug., 2003) to be published.

[8 ] R. A. Hiller and S. J. Putterman, Phys. Rev. LETT., 75, 19, 3549 (1995)

[9] M. P. Brenner, Sascha Hilgenfeldt, and D. Lohse, Single-bubble Sonoluminescence, Rev. of Mod. Phy., 74, 425484, (2002) 
Figure 1

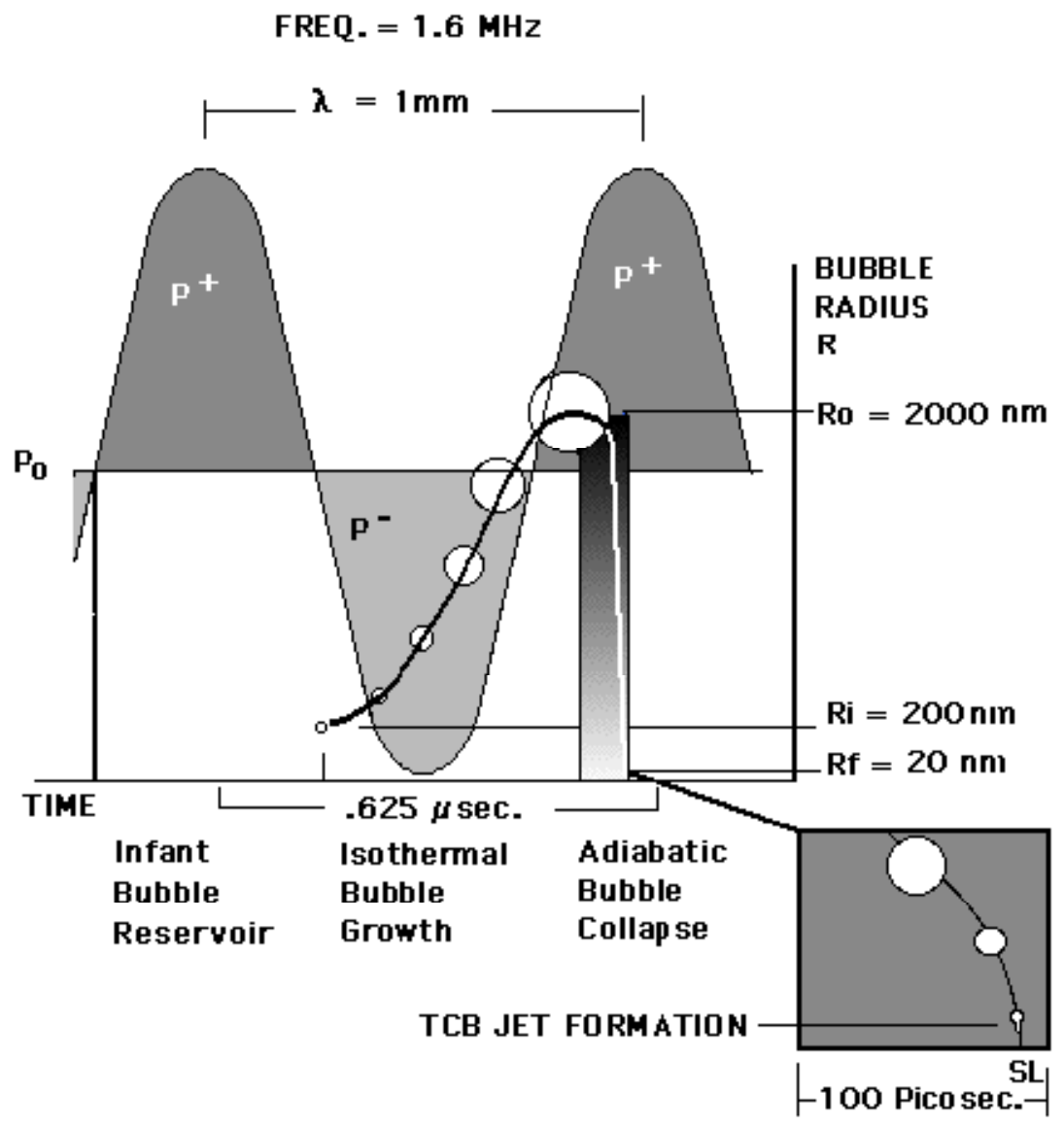

Figure 1 shows a schematic of the growth and collapse of a bubble in an intense $1.6 \mathrm{MHz}$ acoustic field producing TCBs as the acoustic wave progresses through a cycle. The time period for this process is less than 0.6 microseconds. The infant bubble at a radius of Ri originates in the low pressure portion of the oscillating acoustic field where the Ri expands rapidly and isothermally and upon reaching the high pressure portion of the acoustic field the bubble growth slows reaching a maximum radius Ro. At this point, with its newly gained mass, the bubble starts its implosive and accelerated collapse path to radius Rf. Here a portion of the collapse bubble contents which are now dissociated into a low energy high density plasma produce a jet and SL. The SL is the only tool we have that communicates the state of the bubble plasma. This collapse process is considered pseudo adiabatic because much of its original mass is lost at the bubble interface. As the pulse of SL photons created by TCB collapse is emitted, with the formation of a jet, the remaining bubble contents become the high density micro accelerators. Some of these accelerated and z-pinched deuteron or proton ions are implanted into target lattices. These final processes are sub-picosecond in duration. 
Figure 2

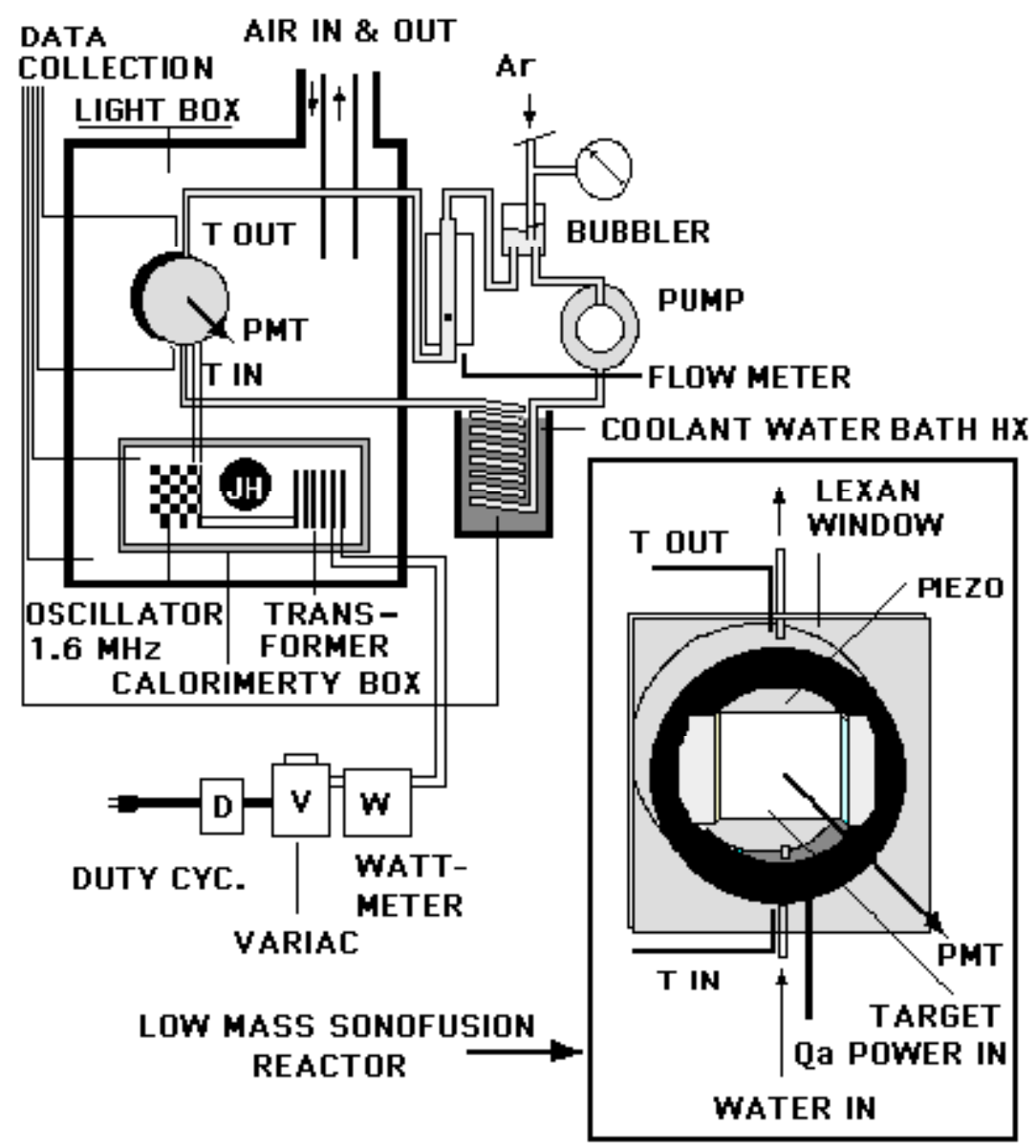

Figure 2 shows the experimental set-up for gathering data from the reactor - lower left inset. It is made up of a circulation system, a power input system, a SL measurement system, a calorimetric system, a calorimeter for the heat loss of the oscillator and transformer, and a data gathering system. The circulation system has the primary task of removing heat from the low mass SF reactor and the measured DT and flow rate for the calorimetry. The circulation system includes a pump, a coolant bath, a one ml LM SF reactor, a flowmeter, and an Ar bubbler. The power input, Qi, had the option of being pulsed or continuous wave cavitation, $\mathrm{D}$, and that power to the wattmeter, $\mathrm{W}$, and the $1.6 \mathrm{MHz}$ LM reactor is controlled by a variac, V. The SL emission is collected by the PMT and counted in a black box environment. The calorimetric system has two parts. The first part is the measurement of the LM SF reactor DT (T out-T in) and the water flow rate in the black box. The second part is the transformer and oscillator heat losses measured in a calorimetry box calibrated with a Joule heater, JH, also located in the black box with its forced air circulation. The set-up for data gathering consists of a thermocouple system measuring the critical temperatures and a calorimetry box for measuring the steadystate temperature of the transformer and oscillator with its Joule heater that provides for the partition of Qi into Qa and Qo\&t. The bubbler keeps the circulating water, Ar saturated and the coolant bath keeps the $\mathrm{T}$ in close to ambient temperature. The data collection system samples the temperature every five seconds. Lower left inset is the reactor detail showing the $1 \mathrm{ml}$ reactor volume with the placement of the target foil in the reactor window for a baffled PMT SL photon emission counting. 
Figure 3

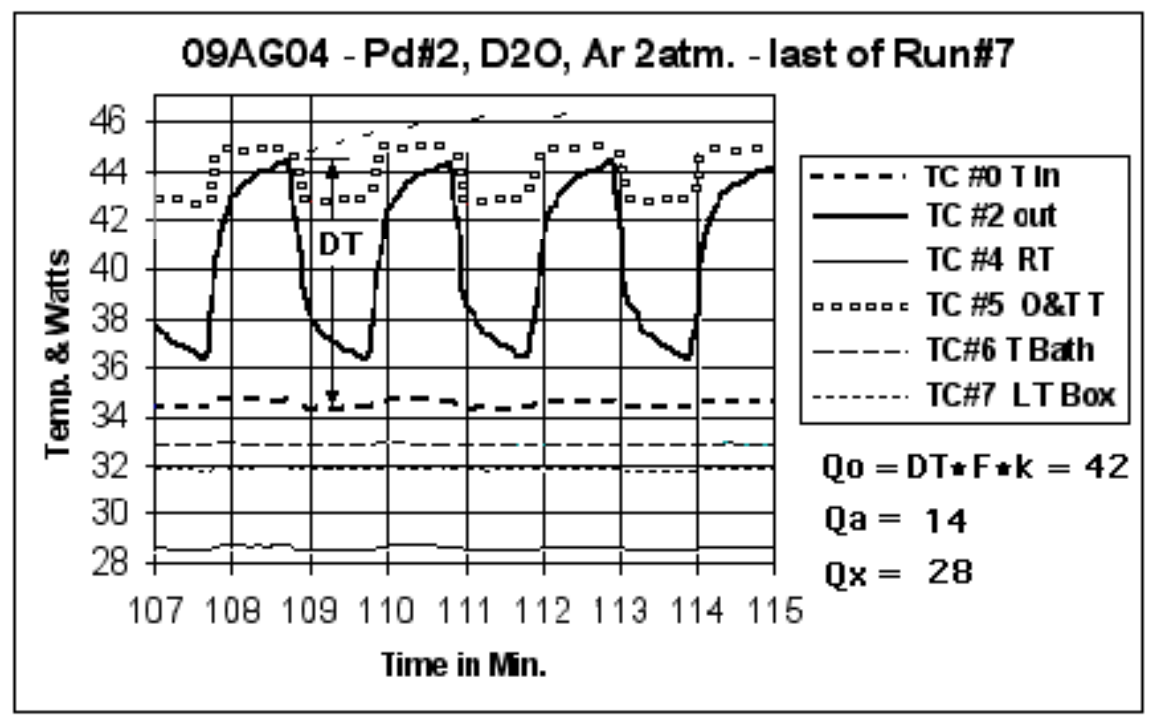

Figure 3 shows an abbreviated form of a data sheet where the data was logged every 5 seconds. This is a data sample of eight minutes near the last of run of Series B cavitated with Pd foil \# 2 as a target, in D2O, and under $2 \mathrm{~atm}$ Ar. This run was typical of the data collected. The first characteristic to note is the shape of the H\&T curves and the one minute duty cycle shown by TC \#2. The second, note the temperature RF interference shown by TC \#0 is no more than $0.3^{\circ} \mathrm{C}$ but the oscillator \& transformer shown by TC $\# 5$ has an RF interference of close to $2.0^{\circ} \mathrm{C}$. Note that the temperature in the light box TC \#7 is constant and does not show RF interference as does the coolant bath temperature, TC \#6. The DT is measured in the off mode and produces a Qo of 42 watts. Qi measured by the wattmeter is partitioned by E to give Qa.

Figure $4 \mathrm{a} \& b$
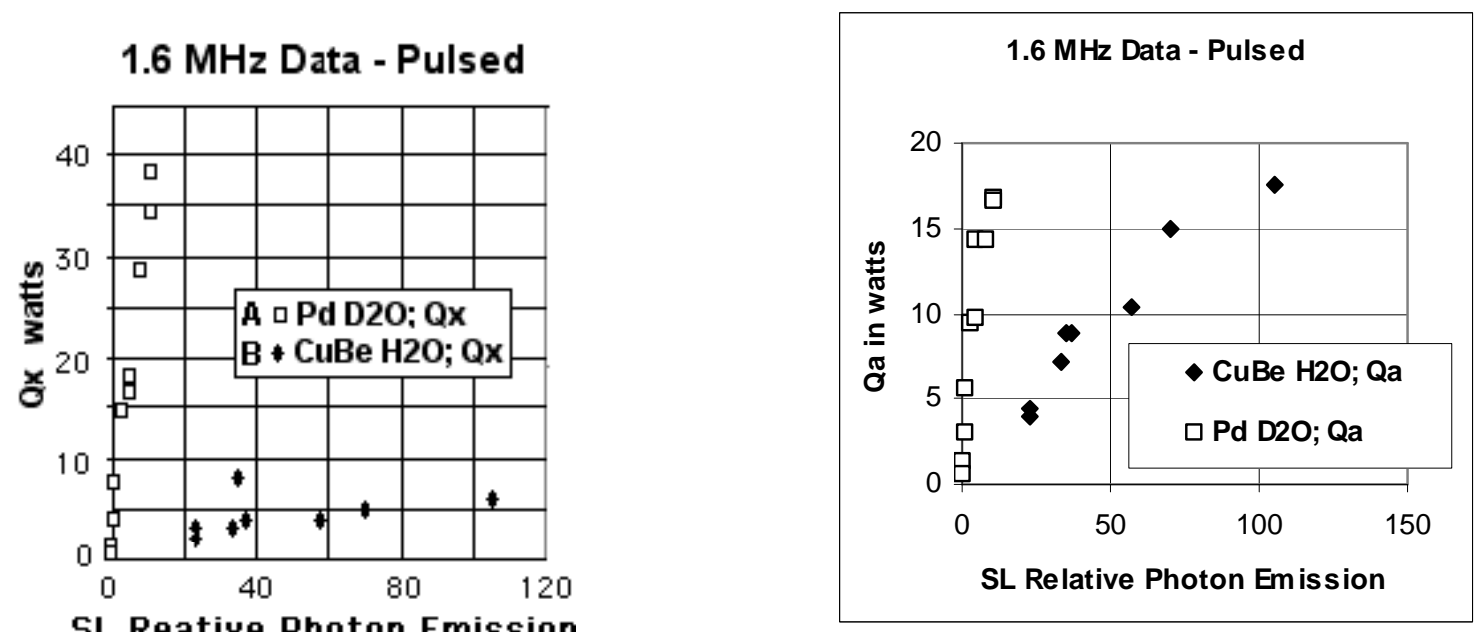

Figures $4 \mathrm{a} \& 4 \mathrm{~b}$ show the pulsed data collected in two different systems; system A, is the Pd target foil with cavitating $\mathrm{D}_{2} \mathrm{O}$ and 1 atm. of $\mathrm{Ar}$ and, system $\mathrm{B}$, is the $\mathrm{CuBe}(98.1 / 1.9)$ with cavitating $\mathrm{H}_{2} \mathrm{O}$ and $1 \mathrm{~atm}$. Ar. The figures $4 \mathrm{a} \& 4 \mathrm{~b}$ 
show the difference between relative intensity of SL (photon emission in 1000 counts/second) with the intensity 10 times greater for $\mathrm{H}_{2} \mathrm{O}$, the $\mathrm{B}$ system as expected [6,7,8]. In figure 4a the excess heat production, Qx, is 8 times greater in $\mathrm{A}$ than $\mathrm{B}$. In figure $4 \mathrm{~b}$ the acoustic input, $\mathrm{Qa}$, does not vary in $\mathrm{A}$ and $\mathrm{B}$ with respect to $\mathrm{D} 2 \mathrm{O}$ and $\mathrm{H} 2 \mathrm{O}$.

Figure $5 \mathrm{a} \& \mathrm{~b}$

Qx Increases With Qa

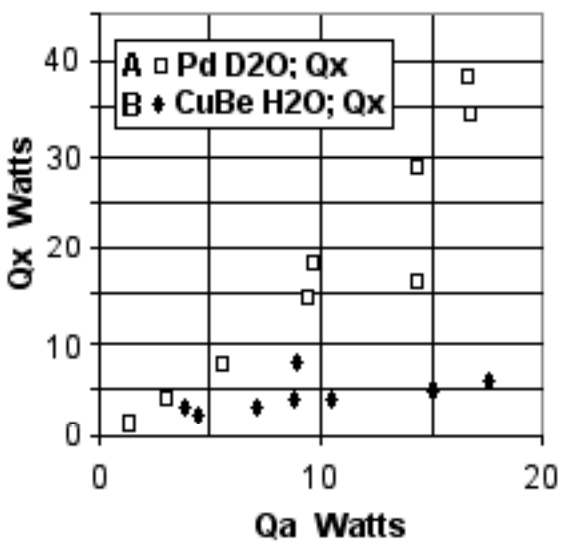

Multiplier Effect, ME

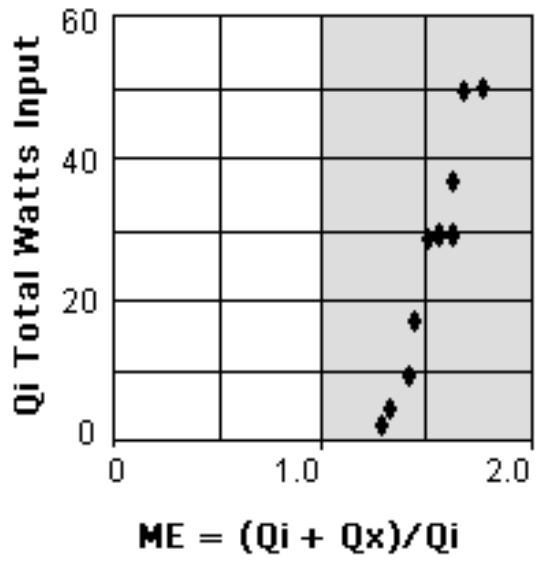

Figure 5a shows that Qx for similar conditions is 2 to 8 times greater with the $\mathrm{D}_{2} \mathrm{O}$ Pd system than the $\mathrm{H}_{2} \mathrm{O} \mathrm{CuBe}$ system. The excess heat increases with the increase of Qa. Figure $5 \mathrm{~b}$ shows the multiplication factor, ME, that indicates the advantage in cost when producing low-grade heat using Qi + Qx to heat water for use as a source for hot water or for space heating in buildings. ME times the power cost - an ME of 2 gives 2 times the power $/ \$$.

Figure 6
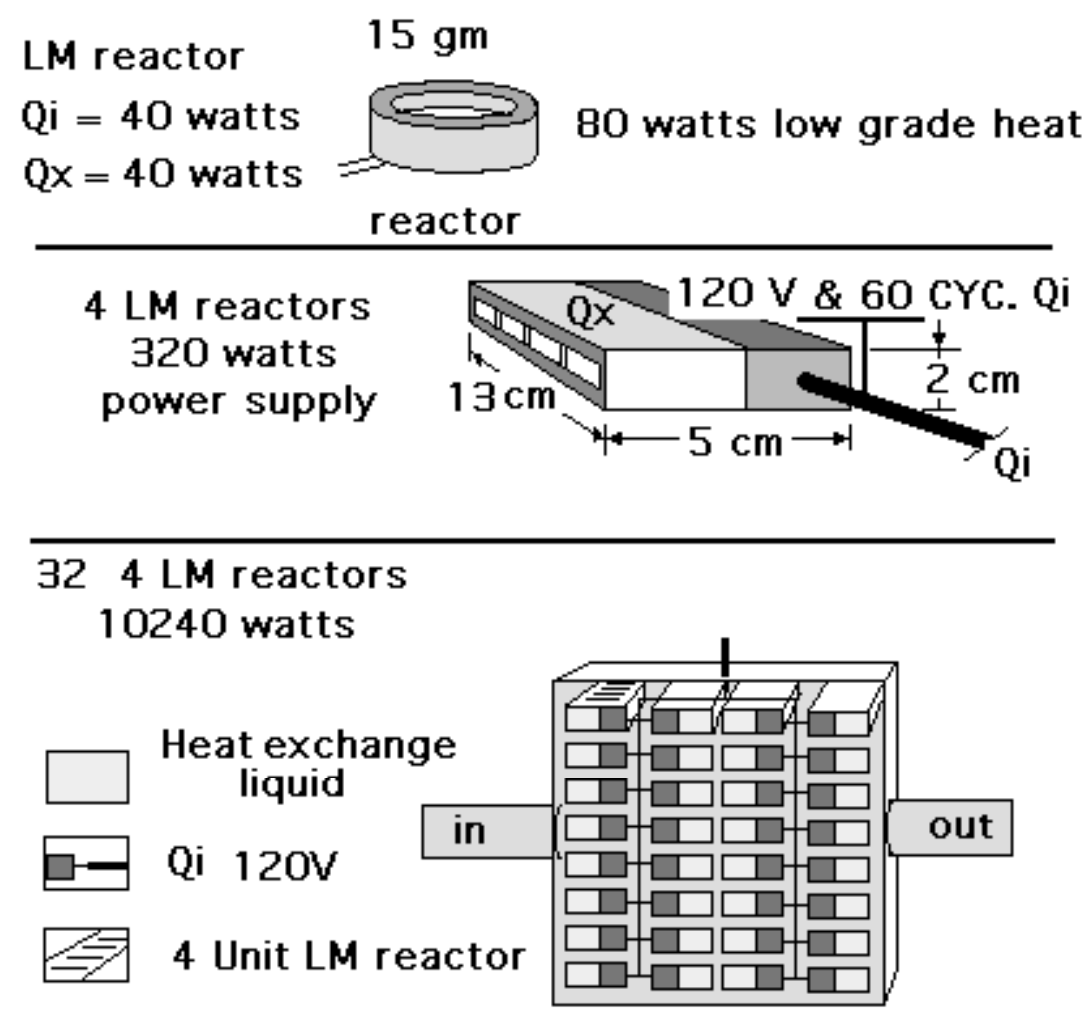
Figure 6 shows a four unit piezo driven LM SF reactor. Ganging 32 them together produces $10 \mathrm{~K}$ watts. Such a device delivers low grade hot water at $80{ }^{\circ} \mathrm{C}$. 